\title{
Diagnostic dilemma and challenges of granulocytic sarcoma: a review
}

\begin{abstract}
The maleficent tumour granulocytic sarcoma is an unusual cancer indite of progressive myeloid precursor cells. It is a Chloroma basically present at an extramedullary location spread through immature granulocytic cells. It can affect any part of the body but the primary site is soft tissues, outside bone marrow, lymph nodes. The root cause may be many factors i.e. genetic, environmental, carcinogens etc. This proliferative type of cancer is sometimes misdiagnosis in contradictory to other acute/chronic tumours. For a histopathologist, it is a challenge for an accurate diagnosis. The tissue biopsy is the known diagnostic technique used since days in cancer diagnosis.

To address this review, our main focus is to investigate, analyse the diagnostic limitations or research gaps in granulocytic sarcoma recognition at the clinical level and what can be the possibilities that have developed recently in tumour detection at an early stage. Next, we will try to develop a hypothesis from pinpoint methods used in it as molecular diagnosis, immune-histochemical biomarkers, flow cytometry (FACS) for confirmation of sarcoma so that specificity and sensitivity can be well examined. The prognosis/diagnosis in myeloid tumour detection depends on key factor innovation and medical devices and that should be filled in research and so a gold standard diagnostic kit should be developed.
\end{abstract}

Volume 9 Issue 4 - 202 I

\author{
Amit Kumar Dubey,' Jagadish Hansa² \\ 'Department of Biotechnology, National Institute of \\ Pharmaceutical Education and Research [NIPER] Hajipur- \\ Vaishali, Bihar, India \\ 2Department of Biotechnology, Radha Govind University, \\ Ramgarh, Jharkhand, India
}

Correspondence: Jagadish Hansa, Department of Biotechnology, Radha Govind University, Ramgarh, Jharkhand, India,Email Jagadish.hansa@gmail.com

Received: April 15, 202I | Published: August 26, 202 I

Keywords: granulocytic sarcoma, diagnosis, histopathology

\section{Introduction}

The green blast chloroma is a histopathological condition called myeloid/granulocytic sarcoma. The green pigment is ascribed due to the enzyme myeloperoxidase. The dissemination of extramedullary tumour blast (soft tissues) is the identification mark of granulocytic cancer. ${ }^{1}$ Cancer that occurs from outside the bone marrow is less than $10 \%$ in acute myeloid leukemias (AML) and most exert influence on dermis/epidermis membrane, lymphoglandula, central sensory system and genitalia etc. This distorts the basic anatomy or physiology of affected tissue. ${ }^{2}$ Sometimes, tumour retrogresses whenever the pluripotent stem cells transplantation surgery of genetically dissimilar tissues of a patient. ${ }^{3}$ Few times it has been seen with chronic myeloid leukemia.

The rare type of sarcoma occurs two in a million in healthy persons when it has seen after the biopsy examination by the clinician. It can affect at any stage of life i.e. juveniles, adults or older persons. This granulocytic tumour hit any area of the human body but the full mechanism is yet to be elucidated. ${ }^{4}$ Further, serious case reports observed at the genito-urinary system in literature search and the treatment is typical in these cases. Therefore, this shows that this disease is infrequent in nature because of its diverse location sites found. If we focus on treatment, then due to limitations in diagnosis, there is not a target-specific therapy but fms-like tyrosine kinase 3 (FLT3) inhibitors used in acute myeloid cancer. ${ }^{5}$

This review put-forth the influence on patients due to misdiagnosed myeloid sarcoma. There is a study published that there are about forty per cent chances of incorrect diagnosis in MS because of the very minute difference between MS and ML (malignant lymphoma) and this wrong diagnosis leads to escalating the symptoms. Further, innovation in medical devices is needed to be prompt to surmount the gap in accurate diagnosis in GS/MS.

\section{Present scenario: diagnostic paradigms}

The specific, sensitive and error-free diagnosis is the initial step in to detect diseases. If we consider granulocytic sarcoma relative to other hematologic lethal cancers, it is easy to diagnosis but the primary myeloid sarcoma is not an easy task to diagnose and different clinical manifestations aroused. ${ }^{6}$ To correct a high sign of hesitancy in MS diagnosis is a challenge for the pathologist. The aetiology difference between granulocytic tumour and malign tumour is highly full of uncertainty; doubtful due to fault in GS/MS diagnosis. ${ }^{7}$ In clinical histology, myeloid sarcoma is allied with AML/CML (acute/ chronic myeloid leukemia), sometimes refers as primary leukemia. ${ }^{8}$ Moreover, another misdiagnosis happens in the cases of cancer of the lymphatic system i.e. T-cell lymphoma (non-Hodgkin's lymphoma), abnormal proliferation of the Langerhans cells i.e. malignant histiocytosis, sarcoma of eosinophils, haematopoietic stem cells cancer at the extramedullary site, a tumour of the Ewing family (TEF) etc. The needle biopsy of bone marrow can be considered nowadays as a preferred method but painful for patients and required handling for a medical practitioner.,

The prime three techniques used in the diagnosis of GS for further evaluation and validation in practices are histopathological/biopsy examination, radiolabelled nucleic acids hybridization methods (In situ-Hybridization/FISH) and Fluorescence-Activated Cell Sorting (FACS/Flow cytometry). ${ }^{11,12}$ Recent advances in genetic engineering can empower the diagnostic approach and can sort out the high index limitation. If the organ system due to sarcoma is at a state of being obstructed then surgery and radiological treatment is options that remains. This isolated/primary granulocytic sarcoma needs special attention in diagnosis because mistreatment further enhances mortality in cancerous patients. ${ }^{13}$

The most often used method in case of soft tissue tumours occur on the surface is ultrasound scan; it measures the dimensions of soft 
tissue mass. ${ }^{14}$ If we consider the synovial sarcoma, an X-ray can be a profound technique for clarification of hardening of tissue/deposition of calcium compounds but more advance specific techniques needed to confirm it. ${ }^{15}$ Both techniques can be used in routine diagnostics. The risk stratification is always there in case of doubtful cases but better technology leads to better diagnosis.

\section{Specific markers in diagnosis}

To segregate the genetically varies tumour cells, the specific markers are the key signal molecules. The sarcoma should be differentiated from lymphoma and leukemia by the expression of the markers profile analysis in clinical samples. The cluster of differentiation (CD) molecules is widely used in diagnosis. Hereby, CD13, CD33, CD43, CD68, CD117 are the prime targets in assays and the enzyme myeloperoxidase through which green lesions presentation in cancer. Whereas other signalling surface antigens such as CD5, CD10, CD11c, CD20, CD34, CD79a, CD99 tumour suppressor/apoptosis regulator $\mathrm{Bcl} 2$ are also to be examined by FACS methods. ${ }^{16-18}$ The anti-macrophage marker antibody MAC387, monoclonal antibody VS38C were also analysed by assays. The high ratio of relapse increases by inappropriate diagnosis and follow up therapy increases if not treated. The prognosis also plays a significant role in differentiated GS. The clinical significance arises more prominently if the signalling pathways mechanism needs to understand in a whole brief way when granulocytic sarcoma cells migrate to the extra-medullary area. For the definitive diagnosis, genetic biomarkers are also needed to verify. A high range of mutations observed in prognostic biomarkers i.e. Ubiquitin Protein Ligase E3 Component N-Recognin 5 gene (UBR5, biomarker gene in cancer) and U2 Small Nuclear RNA Auxiliary Factor 1 gene (U2AF1, biomarker gene in cancer). ${ }^{19,20}$ Others may be Stromal Antigen 2 (STAG2), SAM domain and HD domain-containing protein 1 (SAMHD1), runt-related transcription factor 1 (RUNX1). ${ }^{21}$ The histochemistry (chromagens) markers are determined in the case of a section in sarcoma tissues; one of the A1 markers is Naphthol AS-D chloroacetate esterase. It gives more about $90 \%$ accuracy in diagnosis. The lysozyme enzyme is also isolated in patient's samples and it is also a histochemical biomarker. ${ }^{22}$

\section{Misdiagnosed GS: patient complications}

In the retrogressive literature search, doctors and pathologist find diagnosis is the major hurdle to differentiate between tumours. The referring clinician suggests primely non-Hodgkin's lymphoma whenever the case present at first sight diagnosis. Secondarily, the patients in absentia details of myeloid leukemia are hard to diagnose at first time observation and false-positive reports worsen the symptoms. The amputation of these tumours is not still standardised..$^{23}$ The clinical features should not be well determined. If the patients are not admitted to referral hospitals, the three factors bring out in patient life's complications i.e. poor diagnosis, complex surgeries and mortality. The emerging of new subtypes of sarcoma is also to be tackle in diagnosis as suggested by the world health organisation classification on a tumour in the year 2020, 5 th edition. ${ }^{24}$ The defining features of GS/MS by the WHO are vague and imprecise descriptions so while addressing the patient mislead the diagnostic results. ${ }^{25}$

The diagnosis of myeloid sarcoma is a challenge since from the first case report of a woman was of own kind, it was hard palate GS and published in human pathology in December 1984 and diagnosed with histological findings, and because of delay in diagnosis and treatment, the patient died after the short duration of clinical examination. ${ }^{26}$
If we consider the recently presented case reports, then a 12-year boy died due to delay in analysing the medical situation after the treatment of cancer of B-cell because myeloid sarcoma develops after 8 months post-treatment. High throughput sequencing shows mutations in related oncogenes genes. Even-though stem cell transplantation could not revive healthy tissues. Pathologists were uncertain in this case that it was a case of a primary or secondary outset. Therefore, it is a direct case of delay \& poor diagnosis. ${ }^{27}$

One more case of a fifteen-year teenager $O$; the report was presented by the author in the year 2016, histochemical diagnosis suggested a GS case not a case of malignant lymphoma. The risk factor was high in this case to detection. ${ }^{28}$

\section{To be filled: a gap junction}

In granulocytic sarcoma, prognosis factors need to be verified and mechanism to be validated for precision in diagnosis. There is a big gap in research in histopathological diagnosis and that has to be filled by a big leap in innovation. To lead a better medication and therapy in the cases of GS confirmed cases there should be standard compliance for patients and modern approaches in diagnosis/prognosis. In recent years, researchers and clinician have found and prescribed bio-imaging technology such as computed tomography in the cases of misdiagnosis/ doubtful MS to differentiate the reports of AML. In today's era, high throughput technology or advance radio imaging in biomolecules can only fill a gap in diagnosis to proceed to the right diagnosis and better patient treatments. One more approach has applied positron emission tomography (radio-imaging) technology where radioisotopes applied in diagnosis; the fluoro18deoxyglucose isotope molecule used to detect granulocytic lesions. These $\mathrm{F}^{18} \mathrm{DG}-\mathrm{PET} \mathrm{CT}$ scans have shown excellence to determine tumour's size and area of localisation. There is a clinical trial registered on clinicaltrials.gov where PET-CT used in acute myeloid leukemia for the examination of extra-medullary manifestations. Researchers are also trying to develop advancement in magnetic resonance imaging (MRI) in cases of central nervous systems with suspected tumours. ${ }^{29,30}$

Every cell has a unique expression profile and marker profile and it should be well elucidated and documented in patient history by the methods of pharmacogenomics. Therefore, an advance microarray system can be a defined technology to get tumour-specific expression marker profiling data. With this diagnosis, in addition; the well planned pin-sharp needle biopsy can reverse the adverse outcomes in patients. Next-generation sequencing (NGS) can also a technique that can monitor mutations in genes related to the suspected zone of tumour. ${ }^{31}$ Moving further in better advance diagnostic tools then nanotechnology for the identification of medical images of chemical biomarkers (tagging) and artificial intelligence for the transcriptome and expression profile barcode studies based on the algorithm is the next decade technology to fulfil this gap in GS diagnosis. ${ }^{32,33}$ These technologies will be a robust approach in GS/MS diagnosis.

\section{Future remarks}

The right indorsation diagnosis is foremost to prevent inapt chemotherapy. Indeed, minor points are also considered but still, the proven cases should be documented and standardised by the cancer society. The prevalence, site of occurrence and the prognostic signs for the patient is required when the accuracy prevails in diagnosis and De-nova diagnostic kits to be well-calibrated and applied in GS. The dynamic studies of the live cells in contrast to tumours may be included in diagnosis reports and this will impact on diagnosis. 


\section{Acknowledgments}

The authors acknowledge the support from the National Institute of Pharmaceutical Education and Research, Hajipur, Ministry of Chemicals and Fertilizers, Govt. of India.

\section{Conflicts of interest}

The authors declare no conflict of interest in this work.

\section{References}

1. Cantone E, Cavaliere M, Di Lullo AM, et al. Immunohistochemical patterns in the differential diagnosis of rhinopharyngeal granulocytic sarcoma. Oncology letters. 2016;12(4):2777-2781.

2. Kohli S, Lee M, Marshall S. A case report on the progression of myeloid sarcoma to form multiple metastatic deposits without developing acute myeloid leukaemia. Case reports in hematology. 2015;2015:162154.

3. Navarro AM, Susanto E, Falk A, et al. Modeling cancer using patientderived induced pluripotent stem cells to understand development of childhood malignancies. Cell death discovery. 2018;4(1):1-9.

4. Yilmaz AF, Saydam G, Sahin F, et al. Granulocytic sarcoma: a systematic review. American journal of blood research. 2013;3(4):265.

5. Avni B, Koren-Michowitz M. Myeloid sarcoma: current approach and therapeutic options. Therapeutic advances in hematology. 2011;2(5):309316.

6. Mccusker Scott, Trangucci J, Frederick W, et al. Primary Myeloid Sarcoma of the Small Intestine: Case Report and Literature Review. Connecticut medicine. 2016;80(6):349-352.

7. Pramanik R, Tyagi A, Chopra A, et al. Myeloid sarcoma predicts superior outcome in pediatric AML; can cytogenetics solve the puzzle?. Clinical Lymphoma Myeloma and Leukemia. 2018;18(6):e249-e254.

8. Antic D, Elezovic I, Milic N, et al. Is there a "gold" standard treatment for patients with isolated myeloid sarcoma?. Biomedicine \& Pharmacotherapy. 2013;67(1):72-77.

9. Yamauchi K, Yasuda M. Comparison in treatments of nonleukemic granulocytic sarcoma: report of two cases and a review of 72 cases in the literature. Cancer. 2002;94(6):1739-1746.

10. Bakst RL, Tallman MS, Douer D, et al. How I treat extramedullary acute myeloid leukemia. Blood. 2011;118(14):3785-3793.

11. Park KU, Lee DS, Lee HS, et al. Granulocytic sarcoma in MLL-positive infant acute myelogenous leukemia: fluorescence in situ hybridization study of childhood acute myelogenous leukemia for detecting MLL rearrangement. The American journal of pathology. 2001;159(6):20112016.

12. Seifert RP, Bulkeley III W, Zhang L, et al. A practical approach to diagnose soft tissue myeloid sarcoma preceding or coinciding with acute myeloid leukemia. Annals of diagnostic pathology. 2014;18(4):253-260.

13. Klco JM, Welch JS, Nguyen TT, et al. State of the art in myeloid sarcoma. International journal of laboratory hematology. 2011;33(6):555565 .

14. Wells PN, Liang HD. Medical ultrasound: imaging of soft tissue strain and elasticity. Journal of the Royal Society Interface. 2011;8(64):1521-1549.

15. Di Marzo A, Parca G, Ingrosso G, et al. Retro-orbital granulocytic sarcoma: case report. Tumori Journal. 2008;94(6):869-872.
16. Kaur V, Swami A, Alapat D, et al. Clinical characteristics, molecular profile and outcomes of myeloid sarcoma: a single institution experience over 13 years. Hematology. 2018;23(1):17-24.

17. Chen J, Yanuck III RR, Abbondanzo SL, et al. c-Kit (CD117) reactivity in extramedullary myeloid tumor/granulocytic sarcoma. Archives of pathology \& laboratory medicine. 2001;125(11):1448-1452.

18. Kumar P, Singh H, Khurana N, et al. Diagnostic challenges with intraoral myeloid sarcoma: report of two cases \& review of world literature. Exp Oncol. 2017;39(1):78-85.

19. Jain S, Mazaheri B, Raviv N, et al. Cancer classification from healthy DNA using machine learning. 2019.

20. Koul B. Cancer Genomics. In Herbs for Cancer Treatment. Springer, Singapore. 2019:1-52

21. Burns AD. Applications of next-generation technologies in the diagnosis of haematological diseases and cancer (Doctoral dissertation, Oxford Brookes University). 2016.

22. Braun JD, Géraud C, Marx A, et al. Bilateral Inguinal Ulcerations as First Presentation of Acute Myeloid Leukaemia. Acta dermatovenereologica. 2019;99(11-12):1041-1042.

23. Vodanovich DA, Choong PF. Soft-tissue sarcomas. Indian journal of orthopaedics. 2018;52:35-44.

24. Choi JH, Ro JY. The 2020 WHO classification of tumors of soft tissue: selected changes and new entities. Advances in Anatomic Pathology. 2021;28(1):44-58.

25. Shallis RM, Gale RP, Lazarus HM, et al. Myeloid sarcoma, chloroma, or extramedullary acute myeloid leukemia tumor: A tale of misnomers, controversy and the unresolved. Blood Reviews. 2020;100773.

26. Castella A, Davey FR, Elbadawi A, et al. Granulocytic sarcoma of the hard palate: report of the first ase. Human pathology. 1984;15(12):1190-1192.

27. Yuan X, Yu U, Chen S, et al. Case Report: Myeloid Sarcoma Development During Treatment for B Cell Lymphoblastic Lymphoma in a Boy with KRAS/NRAS Gene Mutations. OncoTargets and therapy. 2021;14:347353.

28. Das H, Hansa J, Sahu MC, et al. Granulocytic sarcoma of cervical lymphnode: A diagnostic challenge. Cancer Treatment Communications. 2016;7:17-20.

29. Stölzel F, Lüer T, Löck S, et al. The prevalence of extramedullary acute myeloid leukemia detected by $18 \mathrm{FDG}-\mathrm{PET} / \mathrm{CT}$ : final results from the Prospective PETAML Trial. Haematologica. 2020;105(6):1552.

30. Muhleman M, Ivanovic M, Khandani AH. PET/CT and PET/MR in Soft Tissue Sarcoma and Melanoma Patients: What to Image and How to Image It. In PET/CT and PET/MR in Melanoma and Sarcoma. Springer, Cham. 2021. pp. 31-77.

31. Desai AN, Jere A. Next-Generation Sequencing for Cancer Biomarker Discovery. In Next Generation Sequencing in Cancer Researc. Volume 2, 2015: pp. 103-125.

32. Ye F, Zhao Y, El-Sayed R, et al. Advances in nanotechnology for cancer biomarkers. Nano Today. 2018;18:103-123.

33. Esterhuysen FN. Development of a simple artificial intelligence method to accurately subtype breast cancers based on gene expression barcodes. 2018 . 